\title{
Factors predicting suitability of organ preservation with radiation therapy in laryngeal and hypopharyngeal cancer
}

\author{
Parul N. Barry¹, Adrianna L. Henson'1, Nicole E. Goodwin ${ }^{2}$, Elizabeth Cash ${ }^{3}$, Megan A. Mezera ${ }^{1}$, Liz \\ Wilson ${ }^{4}$, Rebecca A. Redman ${ }^{5}$, Cesar A. Perez ${ }^{5}$, Jeffrey M. Bumpous ${ }^{4}$, Kevin L. Potts ${ }^{4}$, Craig L. \\ Silverman $^{1}$, Neal E. Dunlap ${ }^{1}$ \\ 1. Departments of Radiation Oncology, University of Louisville, Louisville, United States. 2. University of Louisville, \\ Hospital Speech Pathology, Louisville, United States. 3. Psychological \& Brain Sciences, University of Louisville, Louisville, \\ United States. 4. Surgery-Division of Otolaryngology, University of Louisville, Louisville, United States. 5. Medical \\ Oncology, University of Louisville, Louisville, United States
}

Correspondence: Neal E. Dunlap, MD. Address: 529 S Jackson Street, Louisville, KY 40202, United States.

Email: nedun101@exchange.louisville.edu

Received: October 29, 2014

Accepted: December 4, 2014 Online Published: December 10, 2014

DOI : $10.5430 /$ jst.v4n4p9

URL: http://dx.doi.org/10.5430/jst.v4n4p9

\section{Abstract}

Objective: In the treatment of laryngeal and hypopharyngeal cancer, organ preservation is ideal as it allows for retained swallowing function and improved quality of life. We hypothesize that pretreatment patient and tumor characteristics can be used to create a predictive model to determine which patients will benefit most from organ preservation.

Methods: We performed a retrospective review of a prospectively maintained database for 58 patients with laryngeal and hypopharyngeal cancer treated with radiation therapy at our institution from 2006 to 2013. Patients were evaluated with a modified barium swallow test (MBS) before and after treatment. Additionally three scales were used to describe swallowing function: the Functional Oral Intake Scale (FOIS), the 8-point penetration/aspiration scale, and the Swallowing Performance Status Scale (SPSS). Stepwise regressions were used to explore possible predictors in the following four models: demographics, disease characteristics, treatment characteristics, and initial swallowing function.

Results: Once significant predictors were established, a final predictive model was created controlling for transcutaneous neuromuscular electrical stimulation (TNMES) and pretreatment swallowing scores. Black race $(p=0.016)$ and those with poorer FOIS ( $p=0.002)$, SPSS $(0.007)$, and 8-point $(p=0.014)$ scores at baseline experienced worse swallowing and aspiration outcomes after completion of treatment. Those who were classified as g-tube dependent on the FOIS at baseline remained dependent at follow-up $(p=0.008)$.

Conclusions: Initial swallowing dysfunction, as well as feeding tube placement, is highly predictive of poor swallowing outcome after organ preservation. Additionally, black race may be related to worse outcome.

\section{Key words}

Organ preservation, Swallowing dysfunction, Dysphagia, Laryngeal and hypopharyngeal cancer 


\section{Introduction}

The treatment of laryngeal and hypopharyngeal cancers with definitive radiation therapy is preferred as it allows for laryngeal preservation without compromising survival ${ }^{[1-3]}$. Advancements in radiation treatment technique and delivery have allowed for improved quality of life, although much initial focus has been on preservation of salivary gland function. It is well established that dysphagia is exacerbated with combined chemoradiation therapy and significantly affects quality of life in patients who undergo this treatment for head and neck cancer ${ }^{[4]}$.

The severity of dysphagia can significantly affect one's quality of life and feelings of anxiety and depression as eating is a social activity and provides nutrition ${ }^{[5]}$. Swallowing is a complex process requiring the coordination of multiple muscles and cranial nerves ${ }^{[4,6]}$. During radiation, patients may develop pain, mucositis, radiation dermatitis, and swelling of the soft tissues of the head and neck. Symptomatology months after treatment are mainly due to fibrosis, lymphedema and damage to the swallowing structures. These radiation side effects typically exacerbate swallowing dysfunction. As such, it is recommended that pre and post-treatment swallowing function be evaluated, especially given that many patients with laryngeal and hypopharyngeal cancers have significant pretreatment swallowing dysfunction ${ }^{[7]}$. Limiting dose to dysphagia aspiration risk structures (DARS) using IMRT and rehabilitation with speech therapy may help improve outcomes $^{[8,9]}$.

An internal review of patients treated at our institution showed that definitive chemoradiation exacerbated swallowing dysfunction in patients with head and neck cancer, and that Transcutaneous Neuromuscular Electrical Stimulation (TNMES) therapy allowed for dysphagia reduction with significant improvement in swallowing scores ${ }^{[10]}$. TNMES is a modality used during swallowing therapy. Electrodes are placed in strategic locations on the anterior neck to stimulate muscle contraction with electrical current. The current is biphasic, $80 \mathrm{Hertz}$, and has a maximum setting of $25 \mathrm{~mA}$ to target the fast twitch or type II muscle fibers. The intent is to strengthen the muscle and improve overall function. Given the improvement in swallowing scores with this intervention, we sought to define factors that would predict for suitability of organ preservation in patients who receive radiation therapy, for treatment of their cancer. To determine such factors would allow for better patient selection for radiation therapy, and help us identify who may benefit from early intervention and rehabilitation.

\section{Methods}

After obtaining institutional review board approval from the University of Louisville, we performed a retrospective review of a prospectively maintained database containing 1,820 patients diagnosed with head and neck cancer from 2006 to 2013. Of those, 393 patients were found to have primary laryngeal or hypopharyngeal cancer requiring definitive treatment either with surgery or radiation. For our study, patients were staged using the 2010 AJCC staging criteria. Fifty-eight patients, all of whom were treated at our institution, underwent both pre and post treatment modified barium swallow test (MBS) evaluation and had their complete medical data available to us for analysis. Patients who had a laryngectomy prior to radiation, previous radiation therapy to the head and neck, and recurrent disease were excluded.

All patients underwent an MBS before and after treatment, with the average post treatment evaluation occurring at 3 months (standard deviation 6.1 months). Nine patients underwent their final MBS prior to completion of treatment due to concern for compliance with follow-up. Additionally, three scales were used to describe swallowing function: the Functional Oral Intake Scale (FOIS), the 8-point penetration/aspiration scale, and the Swallowing Performance Status Scale (SPSS). These scales were performed in addition to the patients MBS study. The FOIS score is divided into levels of oral intake. Each level indicates a progression from non-oral/tube dependency to complete oral intake without dietary restrictions. With this scale, the evaluator can rate the patient's functional oral intake. The SPSS also provides progressive ratings for a patient's oral intake, as well as indicates if the patient is aspirating. The advantage of this scale is the ability to indicate minimal oral deficits that may not necessarily require diet modifications. The disadvantage is that this scale is 
very sensitive but not very specific for evaluating if a patient is aspirating. The 8-point scale is better in that sense, as it shows the severity of penetration or aspiration that an examinee may have with a bolus with relation to the timing of the bolus (see Table 1) ${ }^{[10]}$. The speech pathologist has identified cut points for swallowing dysfunction. Those with FOIS scores $\leq 3$ and SPSS scores $\geq 6$ were defined as PEG tube dependent. Those with 8 -point scores $\geq 6$ were noted to have aspiration. These scales are used in conjunction with each other to get the most accurate description of a patient's swallowing function. Within our sample population, we identified patient demographics, disease and treatment characteristics, and swallowing function. ANOVA, stepwise and logistic regressions were performed to identify predictive factors for development of dysphagia.

Table 1. Swallowing Scoring Scales

\begin{tabular}{|c|c|c|c|}
\hline Score & FOIS (1-7) & SPSS (1-7)* & 8-pt PAS (1-8) \\
\hline 1 & No oral intake & Normal swallowing & Material does not enter airway \\
\hline 2 & $\begin{array}{l}\text { Tube dependent with } \\
\text { minimal/inconsistent oral intake }\end{array}$ & Within functional limits & $\begin{array}{l}\text { Material enters the airway, remains above } \\
\text { the vocal folds, and is ejected from the } \\
\text { airway. }\end{array}$ \\
\hline 3 & $\begin{array}{l}\text { Tube supplements with consistent oral } \\
\text { intake }\end{array}$ & Mild impairment & $\begin{array}{l}\text { Material enters the airway, remains above } \\
\text { the vocal folds, and is not ejected from the } \\
\text { airway. }\end{array}$ \\
\hline 4 & $\begin{array}{l}\text { Total oral intake of a single } \\
\text { consistency }\end{array}$ & Mild-moderate impairment & $\begin{array}{l}\text { Material enters the airway, contacts the } \\
\text { vocal folds, and is ejected from the } \\
\text { airway. }\end{array}$ \\
\hline 5 & $\begin{array}{l}\text { Total oral intake of multiple } \\
\text { consistencies requiring special } \\
\text { preparation }\end{array}$ & Moderate impairment & $\begin{array}{l}\text { Material enters the airway, contacts the } \\
\text { vocal folds, and is not ejected from the } \\
\text { airway. }\end{array}$ \\
\hline 6 & $\begin{array}{l}\text { Total oral intake with no special } \\
\text { preparation, but must avoid specific } \\
\text { foods or liquid items }\end{array}$ & Moderate-severe impairment & $\begin{array}{l}\text { Material enters the airway, passes below } \\
\text { the vocal folds, and is ejected into the } \\
\text { larynx or out of the airway. }\end{array}$ \\
\hline 7 & Total oral intake with no restrictions & Severe impairment & $\begin{array}{l}\text { Material enters the airway, passes below } \\
\text { the vocal folds, and is not ejected from the } \\
\text { trachea despite effort. }\end{array}$ \\
\hline 8 & & & $\begin{array}{l}\text { Material enters the airway, passes below } \\
\text { the vocal folds, and no effort is made to } \\
\text { eject. }\end{array}$ \\
\hline
\end{tabular}

Note. FOIS - Functional Oral Intake Scale, SPSS - Swallowing Performance Status Scale, 8-pt PAS - 8 point Penetration-Aspiration Scale, *- Description of individual impairments not been listed here. Bold - Aspirators and Tube-Dependent.

\section{Results}

We evaluated 58 patients with laryngeal and hypopharyngeal cancer treated from 2006 to 2013. The baseline patient and tumor characteristics are listed in Table 2. Average follow-up was 28.5 months (standard deviation of 23.5 months).

To test hypotheses, stepwise regressions were used to explore possible predictors in the following four models: demographics, disease characteristics, treatment characteristics, and initial swallowing function (see Table 2 for specific characteristics examined). These were run separately to help eliminate potential collinearity between predictors. Once significant predictors were established, a final model was developed, which entered number of TNMES treatments and initial swallowing function as control variables, followed by significant predictors of interest, and swallowing function as the outcome variable to determine predictive value of patient and treatment factors. Models were tested for raw scores, and then for dichotomized (PEG tube or aspiration status) scores, separately for each of the three swallowing outcome 
measures. While there is overlap in the range of standard deviations across measurements, this may partly be a result of the reduced range in the measures themselves. Based on Cohen's principles of effect size, or the magnitude of treatment effect, for the statistical analyses suggest medium to large effects and support the validity of results ${ }^{[1]}$.

Table 2. Patient characteristics

\begin{tabular}{|c|c|c|}
\hline Characteristic & & No. (\%) \\
\hline \multirow{2}{*}{ Age (years) } & Median & 58 \\
\hline & Range & $36-82$ \\
\hline \multirow[t]{2}{*}{ Sex } & Male & $44(75.9)$ \\
\hline & Female & $14(24.1)$ \\
\hline \multirow[t]{3}{*}{ Race } & White & $46(79.3)$ \\
\hline & Black & $11(19)$ \\
\hline & Nonwhite, Non black & $1(1.7)$ \\
\hline \multirow[t]{3}{*}{ Insurance } & Self pay & $2(3.4)$ \\
\hline & Medicare/Medicaid & $27(46.6)$ \\
\hline & Private Insurance/ Medicare + Private supplement & $29(50.0)$ \\
\hline \multirow[t]{4}{*}{ County } & Urban Appalachian & $0(0)$ \\
\hline & Urban Non-Appalachian & $50(86.2)$ \\
\hline & Rural Appalachian & $0(0)$ \\
\hline & Rural Non-Appalachian & $2(6.4)$ \\
\hline \multirow[t]{2}{*}{ Primary Site } & Larynx & $37(63.8)$ \\
\hline & Hypopharynx & $21(36.2)$ \\
\hline \multirow[t]{4}{*}{ Tumor Stage } & $\mathrm{T} 1$ & $2(3.4)$ \\
\hline & $\mathrm{T} 2$ & $20(34.5)$ \\
\hline & $\mathrm{T} 3$ & $28(48.3)$ \\
\hline & $\mathrm{T} 4$ & $8(13.8)$ \\
\hline \multirow[t]{2}{*}{ Nodal Disease } & Present & $32(55.2)$ \\
\hline & Absent & $26(44.8)$ \\
\hline \multirow[t]{5}{*}{ AJCC Stage } & I & $1(1.7)$ \\
\hline & II & $12(20.7)$ \\
\hline & III & $16(27.6)$ \\
\hline & IVa & $25(43.1)$ \\
\hline & $\mathrm{IVb}$ & $4(6.9)$ \\
\hline \multirow[t]{3}{*}{$\mathrm{HPV} / \mathrm{P} 16$} & Positive & $5(8.6)$ \\
\hline & Negative & $18(31.0)$ \\
\hline & Not performed/ Results Unavailable & $35(60.3)$ \\
\hline \multirow[t]{2}{*}{ Chemotherapy } & Yes & $48(82.8)$ \\
\hline & No & $10(17.2)$ \\
\hline \multirow[t]{3}{*}{ Radiation Technique } & $3 \mathrm{D}$ & $38(65.5)$ \\
\hline & IMRT & $18(31.0)$ \\
\hline & 3D and IMRT & $2(3.4)$ \\
\hline \multirow[t]{2}{*}{ Fractionation } & Conventional & $20(34.5)$ \\
\hline & Altered & $38(65.5)$ \\
\hline
\end{tabular}

After controlling for the number of TNMES visits, black race was found to be significantly associated with worse swallowing outcomes after treatment $(p=0.016)$ (see Figure 1, Table 3). Those who were classified on the FOIS as g-tube dependent at baseline remained dependent at follow-up $(p=0.008)$. Additionally, those with poorer FOIS $(p=0.002)$, SPSS 
(0.007), and 8-point ( $p=0.014)$ scores at baseline experienced worse swallowing and aspiration outcomes after completion of treatment (see Figure 2, Table 3). After adjusting for number of TNMES treatments and baseline 8-pt aspiration score, negative nodal status remained a significant predictor of aspiration at follow-up $(p=0.048)$ (see Figure 3 , Table 3 ).

Figure 1. Black race was significantly associated with poorer swallowing outcomes after treatment $(p=0.016)$.

Figure 2. Swallowing dysfunction evaluated using the FOIS, SPSS, and 8-Point Scales. Patients with dysfunctional swallowing at baseline were found to do worse in follow-up. (FOIS $p=0.002$, SPSS $p=0.007$, and 8-Point $p=0.014$ ).
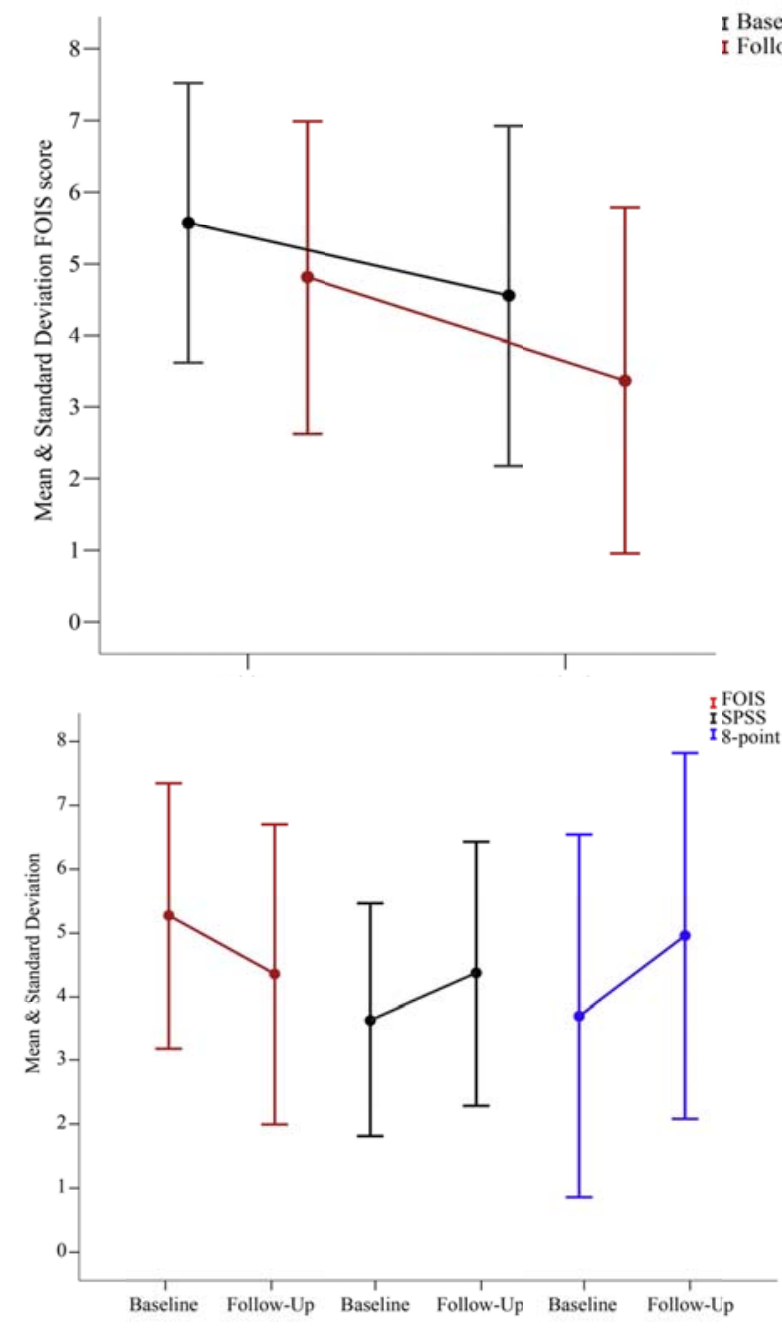

Table 3. Predictors for swallowing dysfunction post-treatment

\begin{tabular}{|c|c|c|c|c|}
\hline & Beta & Partial $r$ & Odds Ratio (95\% CI) & $p$ \\
\hline \multicolumn{5}{|l|}{ FOIS } \\
\hline Race & -0.310 & -0.317 & & 0.016 \\
\hline FOIS baseline & 0.406 & 0.406 & & 0.002 \\
\hline \multicolumn{5}{|l|}{ FOIS tube dependence } \\
\hline FOIS tube dependence baseline & -2.060 & & $7.848(1.733-35.554)$ & 0.008 \\
\hline \multicolumn{5}{|l|}{ SPSS } \\
\hline SPSS baseline & 0.352 & 0.352 & & 0.007 \\
\hline \multicolumn{5}{|l|}{ 8-point } \\
\hline 8-point baseline & 0.321 & 0.321 & & 0.014 \\
\hline \multicolumn{5}{|l|}{ 8-point aspiration } \\
\hline Nodal status & 1.379 & & $3.563(1.183-10.731)$ & 0.048 \\
\hline
\end{tabular}

Note. Partial $r$ is the amount of variance explained after variance explained by control variables (e.g., number of treatment visits, baseline status) was accounted for. Odds ratios are only calculated for models with dichotomous outcomes (e.g., tube dependent versus not). 
Figure 3. Node negativity significantly predicted for increased aspiration as noted on the 8-Point Penetration/Aspiration Scale $(p=0.048)$.

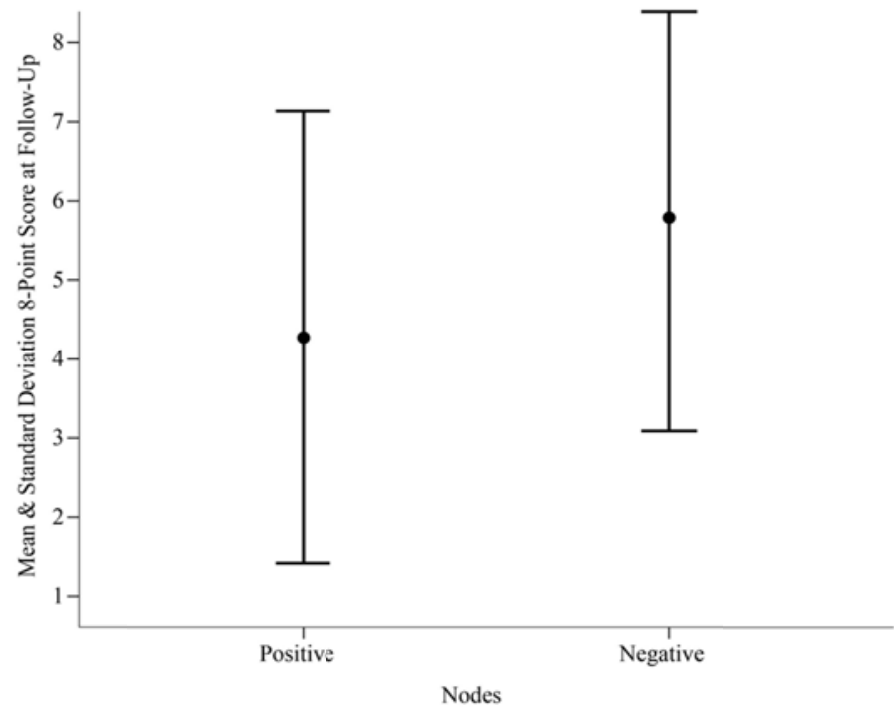

In this cohort, both HPV/P16 positive and negative patients had large tumors. 55.6\% of HPV/P16 negative patients, and $80 \%$ of HPV/P16 positive patients had T3 or T4 tumors. There was no relationship noted between viral status and 8-point aspiration scores at baseline $(p=0.964)$ or follow-up $(p=0.692)$. All black patients in this study had HPV negative disease. However, given the small number of black patients in this study, we were unable to associate their HPV status with their swallowing dysfunction.

Models testing for increased risk of swallowing dysfunction seen on the FOIS, 8-point, and SPSS measures for patients who underwent radiation therapy with either conventional or altered fractionation were non-significant $(p>0.05)$. Similarly, swallowing dysfunction was independent of treatment technique with either 3D conformal radiation therapy or IMRT $(p>0.05)$.

ANOVA and logistic regression models were adjusted for baseline FOIS scores and number of TNMES visits tested for these hypotheses, and all were found to be non-significant $(p>0.05)$. Insurance status and urban or rural residence were not found to significantly predict swallowing dysfunction $(p>0.05)$. Holm-Bonferroni corrections were applied to correct for family-wise error rate present when multiple comparisons are made. All results, except the finding that 8-point scores predict worse swallowing/aspiration outcomes after treatment, remained significant.

\section{Discussion}

Organ preservation in the treatment of laryngeal and hypophargyneal cancer is preferred and allows for improved quality of life. However, development of dysphagia can significantly affect one's quality of life. As such, we sought to determine pretreatment patient characteristics to predict for whom organ preservation would be most appropriate.

Predictors for development of dysphagia after treatment of head and neck cancer with radiation have been evaluated in previous studies. RTOG performed an analysis evaluating severe late toxicity in those who received concurrent chemoradiation for locally advanced head and neck cancer ${ }^{[12]}$. Older age, advanced $\mathrm{T}$ stage, and laryngeal/ hypopharyngeal primary site of disease were found to be independent risk factors for development of late toxicity which included feeding tube dependence, laryngeal/pharyngeal dysfunction, death, infection, and fistula. Neck dissection after chemoradiation was found to exacerbate such toxicities. Also, in a recent update for RTOG 9003, it was noted that disease free patients had lower feeding tube rates when compared to all patients, implying that disease recurrence likely results in 
increased dysphagia ${ }^{[13]}$. Disease-free patients who were treated with an altered fractionation schema, specifically continuous accelerated fractionation, had higher feeding tube usage rates.

In our study, we found that patients with poorer pretreatment characteristics, including g-tube dependence and poorer swallowing scores at baseline, had a worse functional status post-treatment. Additionally, negative nodal stage correlated with poorer swallowing function post-treatment. This finding appears to be a statistical anomaly as We know that in advanced nodal stage patients, there is a higher pharyngo-esophageal structure dose, which has been shown to correlate with increased feeding tube requirement and dysphagia ${ }^{[14]}$. Further analysis is recommended.

In our study, black patients were more likely to have worse swallowing outcomes. Time and again, it has been shown that racial disparities exist, and black patients experience worse clinical outcomes when compared to their white counterparts. A SEER analysis published in 2011 showed that black patients with head and neck cancer experienced increased mortality when compared to whites ${ }^{[15]}$. However, data from the University of Chicago suggests that black patients can experience similar outcomes to whites when treated at centers that treat a large number of minority patients as these patients have increased treatment compliance ${ }^{[16]}$. Additionally, it has been shown that when compared to white patients, black patients find their communication with their physician to be less supportive, less partnering, and less informative ${ }^{[17]}$. Improving such communication will likely increase patient compliance with treatment and with swallowing rehabilitation. Due to the low number of black patients in our patient cohort, our results are hypothesis generating and should be explored further.

Acute and long-term dysphagia can result in nutritional deficiencies, aspiration, feeding tube dependence, and psychosocial morbidity. Clinicians should also keep in mind that dysphagia can result in significant psychosocial morbidity, such as anxiety and depression ${ }^{[5]}$. One study showed that female patients' quality of life is especially affected by their swallowing dysfunction ${ }^{[18]}$. As such, much importance should be placed in prevention of dysphagia. Avoiding and reducing dose to swallowing structures during treatment planning has been evaluated and can reduce prolonged g-tube dependence and improve long term swallowing outcomes. Certain structures in particular are felt to be at risk and include the medial pharyngeal constrictors, glottis and supraglottic larynx, and esophageal inlet. With modern day IMRT, it has been shown that reducing the dose to such organs can reduce swallowing dysfunction ${ }^{[19-21]}$. In our patient population, IMRT plans attempted to spare these structures at risk. However our ability to spare these structures was limited when treating with 3D conformal radiotherapy, which was used in a majority of our patients.

Unfortunately, swallowing dysfunction is typically underreported. This is due in part to the fact that many patients do not undergo swallowing evaluations and that head and neck cancer patients present with multiple symptoms making it is easy to overlook swallowing dysfunction. Ideally, prior to treatment, clinicians should evaluate patient pretreatment characteristics such as g-tube dependence and poor baseline swallowing scores. We recommend that patients undergo a baseline swallowing assessment prior to and after treatment by a certified speech pathologist to identify areas of concern. This will allow for clinicians to assess who will benefit from aggressive prevention and early intervention to attenuate the effects of dysphagia and its sequelae including aspiration and feeding tube dependence ${ }^{[4,22,23]}$.

Organ preservation in larynx and hypopharynx cancer is an appropriate treatment option in a select group of patients. Based upon the results of our review, physicians should take into account their patient's initial swallowing function and feeding tube requirements, due to the finding that baseline swallowing dysfunction correlates with worse functional outcome. Additionally, clinicians should take care to thoroughly communicate the risks and benefits of organ preservation with all of their patients, especially their black patients, as many perceive poorer communication with their physicians. Tumor control and larynx preservation are important, however, predictors for functional outcome, especially dysphagia, should be strongly considered as they can greatly affect one's quality of life. 


\section{Conclusion}

Pre-treatment swallowing dysfunction correlates with a worse functional outcome independent of other tumor or treatment factors. Patients should be evaluated in a multidisciplinary setting to determine if they are better suited for surgical resection with a laryngectomy rather than larynx preservation techniques.

\section{Competing interests}

The authors declare that they have no competing interests.

\section{References}

[1] Induction chemotherapy plus radiation compared with surgery plus radiation in patients with advanced laryngeal cancer. The Department of Veterans Affairs Laryngeal Cancer Study Group. The New England journal of medicine. 1991; 324(24): 1685-90. 2034244. http://dx.doi.org/10.1056/NEJM199106133242402

[2] Lefebvre JL, Andry G, Chevalier D, Luboinski B, Collette L, Traissac L, et al. Laryngeal preservation with induction chemotherapy for hypopharyngeal squamous cell carcinoma: 10-year results of EORTC trial 24891. Annals of oncology: official journal of the European Society for Medical Oncology/ESMO. 2012; 23(10): 2708-14. 22492697.

http://dx.doi.org/10.1093/annonc/mds065

[3] Forastiere AA, Zhang Q, Weber RS, Maor MH, Goepfert H, Pajak TF, et al. Long-term results of RTOG 91-11: a comparison of three nonsurgical treatment strategies to preserve the larynx in patients with locally advanced larynx cancer. Journal of clinical oncology: official journal of the American Society of Clinical Oncology. 2013; 31(7): 845-52. 23182993; PubMed Central PMCID: MC3577950. http://dx.doi.org/10.1200/JCO.2012.43.6097

[4] Rosenthal DI, Lewin JS, Eisbruch A. Prevention and treatment of dysphagia and aspiration after chemoradiation for head and neck cancer. Journal of clinical oncology: official journal of the American Society of Clinical Oncology. 2006; 24(17): 2636-43. 16763277. http://dx.doi.org/10.1200/JCO.2006.06.0079

[5] Nguyen NP, Frank C, Moltz CC, Vos P, Smith HJ, Karlsson U, et al. Impact of dysphagia on quality of life after treatment of headand-neck cancer. International journal of radiation oncology, biology, physics. 2005; 61(3): 772-8. 15708256. http://dx.doi.org/10.1016/j.ijrobp.2004.06.017

[6] Goldsmith T. Videofluoroscopic eval of oropharyngeal swallowing. Mosby, editor. 2003.

[7] Stenson KM, MacCracken E, List M, Haraf DJ, Brockstein B, Weichselbaum R, et al. Swallowing function in patients with head and neck cancer prior to treatment. Archives of otolaryngology--head \& neck surgery. 2000; 126(3): 371-7. 10722011.

[8] Eisbruch A, Schwartz M, Rasch C, Vineberg K, Damen E, Van As CJ, et al. Dysphagia and aspiration after chemoradiotherapy for head-and-neck cancer: which anatomic structures are affected and can they be spared by IMRT? International journal of radiation oncology, biology, physics. 2004; 60(5): 1425-39. 15590174. http://dx.doi.org/10.1016/j.ijrobp.2004.05.050

[9] Roe JW, Carding PN, Dwivedi RC, Kazi RA, Rhys-Evans PH, Harrington KJ, et al. Swallowing outcomes following Intensity Modulated Radiation Therapy (IMRT) for head \& neck cancer - a systematic review. Oral oncology. 2010; 46(10): 727-33. 20850370. http://dx.doi.org/10.1016/j.oraloncology.2010.07.012

[10] Bhatt AD, Goodwin N, Cash E, Bhatt G, Silverman CL, Spanos WJ, et al. Impact of Transcutaneous Neuromuscular Electrical Stimulation on dysphagia in head and neck cancer patients treated with definitive chemoradiation. Head \& neck. 2014. 24710791. http://dx.doi.org/10.1002/hed.23708

[11] Cohen J. Statistical power analysis for the behavioral sciences. 2nd ed: Lawrence Eribaum Associates; 1988.

[12] Machtay M, Moughan J, Trotti A, Garden AS, Weber RS, Cooper JS, et al. Factors associated with severe late toxicity after concurrent chemoradiation for locally advanced head and neck cancer: an RTOG analysis. Journal of clinical oncology: official journal of the American Society of Clinical Oncology. 2008; 26(21): 3582-9. 18559875. http://dx.doi.org/10.1200/JCO.2007.14.8841

[13] Beitler JJ, Zhang Q, Fu KK, Trotti A, Spencer SA, Jones CU, et al. Final results of local-regional control and late toxicity of RTOG 9003: a randomized trial of altered fractionation radiation for locally advanced head and neck cancer. International journal of radiation oncology, biology, physics. 2014; 89(1): 13-20. 24613816. http://dx.doi.org/10.1016/j.ijrobp.2013.12.027

[14] Fua TF, Corry J, Milner AD, Cramb J, Walsham SF, Peters LJ. Intensity-modulated radiotherapy for nasopharyngeal carcinoma: clinical correlation of dose to the pharyngo-esophageal axis and dysphagia. International journal of radiation oncology, biology, physics. 2007; 67(4): 976-81. 17234360. http://dx.doi.org/10.1016/j.ijrobp.2006.10.028 
[15] Rose BS, Jeong JH, Nath SK, Lu SM, Mell LK. Population-based study of competing mortality in head and neck cancer. Journal of clinical oncology: official journal of the American Society of Clinical Oncology. 2011; 29(26): 3503-9. 21844503. http://dx.doi.org/10.1200/JCO.2011.35.7301

[16] Liu GF, Ranck MC, Solanki AA, Cao H, Kolokythas A, Wenig BL, et al. Racial parities in outcomes after radiotherapy for head and neck cancer. Cancer. 2014; 120(2): 244-52. 24122486. http://dx.doi.org/10.1002/cncr.28417

[17] Gordon HS, Street RL, Jr., Sharf BF, Kelly PA, Souchek J. Racial differences in trust and lung cancer patients' perceptions of physician communication. Journal of clinical oncology: official journal of the American Society of Clinical Oncology. 2006; 24(6): 904-9. 16484700. http://dx.doi.org/10.1200/JCO.2005.03.1955

[18] de Campos RJ, Palma PV, Leite IC. Quality of life in patients with dysphagia after radiation and chemotherapy treatment for head and neck tumors. Journal of clinical and experimental dentistry. 2013; 5(3): e122-7. 24455066; PubMed Central PMCID: MC3892254. http://dx.doi.org/10.4317/jced.51092

[19] Li B, Li D, Lau DH, Farwell DG, Luu Q, Rocke DM, et al. Clinical-dosimetric analysis of measures of dysphagia including gastrostomy-tube dependence among head and neck cancer patients treated definitively by intensity-modulated radiotherapy with concurrent chemotherapy. Radiation oncology. 2009; 4: 52. 19909531; PubMed Central PMCID: MC2785826. http://dx.doi.org/10.1186/1748-717X-4-52

[20] Caudell JJ, Schaner PE, Desmond RA, Meredith RF, Spencer SA, Bonner JA. Dosimetric factors associated with long-term dysphagia after definitive radiotherapy for squamous cell carcinoma of the head and neck. International journal of radiation oncology, biology, physics. 2010; 76(2): 403-9. 19467801. http://dx.doi.org/10.1016/j.ijrobp.2009.02.017

[21] Eisbruch A, Kim HM, Feng FY, Lyden TH, Haxer MJ, Feng M, et al. Chemo-IMRT of oropharyngeal cancer aiming to reduce dysphagia: swallowing organs late complication probabilities and dosimetric correlates. International journal of radiation oncology, biology, physics. 2011; 81(3): e93-9. 21592678; PubMed Central PMCID: MC3158965. http://dx.doi.org/10.1016/j.ijrobp.2010.12.067

[22] Mittal BB, Pauloski BR, Haraf DJ, Pelzer HJ, Argiris A, Vokes EE, et al. Swallowing dysfunction--preventative and rehabilitation strategies in patients with head-and-neck cancers treated with surgery, radiotherapy, and chemotherapy: a critical review. International journal of radiation oncology, biology, physics. 2003; 57(5): 1219-30. 14630255.

[23] Murphy BA, Gilbert J. Dysphagia in head and neck cancer patients treated with radiation: assessment, sequelae, and rehabilitation. Seminars in radiation oncology. 2009; 19(1): 35-42. 19028344. http://dx.doi.org/10.1016/j.semradonc.2008.09.007 